\title{
Peers als Quelle aktueller Motivation?
}

\author{
Wie Jugendliche mit heterogenen Verhaltensweisen \\ Peerinteraktionen in Abhängigkeit des behavioralen \\ Klassenumfeldes erleben
}

\author{
Margarita Knickenberg ${ }^{1}$, Carmen Zurbriggen² und Philipp Schmidt ${ }^{1}$ \\ ${ }^{1}$ Fakultät für Erziehungswissenschaft, Universität Bielefeld \\ ${ }^{2}$ Fakultät für Geisteswissenschaften, Erziehungswissenschaften und Sozialwissenschaften, Universität Luxemburg
}

\begin{abstract}
Zusammenfassung: Die Relevanz von Peers für die Entwicklung von Jugendlichen konnte in verschiedenen Studien bereits bestätigt werden. Im Unterricht zeigt sich dies u.a. durch höhere situative Motivation und Freude sowie geringerem Stressempfinden während sozialer Interaktionen mit Mitschülerinnen und -schülern verglichen mit Einzelarbeitssituationen. Allerdings stellte sich auch heraus, dass Jugendliche mit Verhaltensauffälligkeiten Schwierigkeiten im Knüpfen und Aufrechterhalten von sozialen Kontakten und Freundschaften haben. Während in bisherigen Studien Verhalten als dichotome Variable untersucht wurde, wird hier ein dimensionaler, personzentrierter Ansatz zur Identifizierung von Gruppen mit heterogenen Verhaltensweisen verfolgt. Ziel dieses Beitrags ist daher die Untersuchung von Effekten sozialer Interaktionen im Unterricht auf das emotionale Erleben von Jugendlichen mit heterogenen Verhaltensweisen. Zudem wird ein möglicher Moderationseffekt der Klassenkomposition in Bezug auf das Verhalten überprüft. Hierzu wurden N = 719 Schülerinnen und Schülern der fünften und sechsten Jahrgangsstufe unter Verwendung der experience sampling method zu zwei bis drei Messzeitpunkten pro Tag an fünf aufeinanderfolgenden Schultagen zu ihrem aktuellen emotionalen Erleben und ihrem sozialen Kontext befragt. Insgesamt lagen 8870 „Momentaufnahmen“ des emotionalen Erlebens und von Unterrichtssituationen vor. Auf der Grundlage von Verhaltenseinschätzungen durch Lehrpersonen wurden mittels latenter Profilanalysen drei Profiltypen innerhalb der Schülerschaft identifiziert: Jugendliche mit adaptivem, internalisierendem und externalisierendem Verhalten. Ergebnisse von Multilevel-Strukturgleichungsmodellen zeigen, dass Jugendliche in Unterrichtssituationen, in denen sie mit ihren Peers interagieren (z. B. in Partner- oder Gruppenarbeiten), motivierter und weniger gestresst sind als in Einzelsituationen. Für Jugendliche mit externalisierendem oder internalisierendem Verhalten ist dieser Effekt stärker ausgeprägt. Die geringfügigen, jedoch nicht signifikanten differenziellen moderierenden Effekte verweisen zudem darauf, dass der Zusammenhang des emotionalen Erlebens und der Peerinteraktionen für Jugendliche nicht durch ihr Klassenumfeld in Bezug auf prosoziales oder problematisches Verhalten moderiert wird. Die Ergebnisse werden sowohl methodisch als auch inhaltlich diskutiert, unter anderem in Bezug auf die Frage, ob und inwiefern Peers Quellen aktueller Motivation im Unterricht darstellen.
\end{abstract}

Schlüsselwörter: Emotionales Erleben, soziale Interaktionen, Peers, Klassenkomposition, internalisierendes und externalisierendes Verhalten

Peers as a source of current academic motivation? - How students with heterogeneous behaviour experience peer interactions in relation to their classroom environment regarding behaviour

Abstract: The relevance of peers for adolescents' development has already been confirmed in various studies. Among other things, adolescents indicated higher situational motivation and joy as well as less feelings of stress during social interactions with classmates compared to individual work settings. However, it turned out that adolescents with behavioural problems have difficulties in making and maintaining social contacts and friendships. While in previous studies behaviour was investigated as dichotomous variable, this study chose a dimensional, person-centered approach to identify adolescents with heterogeneous behaviour. Therefore, this paper aims at analysing effects of social interactions at school on emotional experiences of adolescents with heterogeneous behavioural profiles. In addition, a potential moderating effect of the classroom environment regarding behaviour will be tested. The sample comprised $N=719$ students of grade five and six. Data were collected using the experience sampling method. In detail, the students completed a short questionnaire concerning their momentary emotional experience and their social context two to three times a day on five consecutive days at school. Thus, 8870 snapshots of the adolescents' emotional experiences and situations in class were available. Using teacher ratings regarding the students' behaviour, latent profile analyses were calculated and three different profiles within the sample were identified: students with adaptive, with internalising and with externalising behaviour. Findings of multilevel structural equation models show, first, that students are more motivated and less stressed while interacting with others during lessons (e.g. in partner or group activities) compared to individual working situations. This effect is more pronounced for students with internalising and externalising behavioural profiles. Second, the small differential moderating, but not significant effects indicate that the relation between adolescents' emotional experiences and peer interactions is not moderated by the classroom environment 
regarding prosocial or problem behaviour. The results will be discussed from methodological and content-related perspectives.

Keywords: Emotional experiences, social interactions, peers, classroom composition, internalising and externalising behaviour

Das emotionale Erleben von Schülerinnen und Schülern steht in einem engen Zusammenhang mit ihrem Verhalten sowie ihren motivationalen Merkmalen. Dementsprechend haben im Unterricht erlebte Emotionen nicht nur eine große Bedeutung für das schulische Wohlbefinden, sondern auch für die Lern-, Leistungs- und Persönlichkeitsentwicklung (Dai \& Sternberg, 2004). Da das emotionale Erleben sowohl kontext- als auch situationsabhängig ist (Bolger \& Laurenceau, 2013; Pekrun \& LinnenbrinkGarcia, 2014), können dadurch Informationen über die quality of experience bzw. Erlebensqualität verschiedener schulischer Unterrichtssituationen gewonnen werden (Venetz, Tarnutzer, Zurbriggen \& Sempert, 2012): So zeigen Ergebnisse von Untersuchungen beispielsweise, dass Jugendliche in Unterrichtssituationen, in denen sie die Möglichkeit haben, mit ihren Mitschülerinnen und Mitschülern zu arbeiten, im Allgemeinen motivierter und weniger gestresst sind als in Einzelarbeitssituationen (Zurbriggen \& Venetz, 2016). Eine stärkere Kontextabhängigkeit des emotionalen Erlebens kann für Jugendliche mit Verhaltensauffälligkeiten (im Vergleich zu Jugendlichen ohne Verhaltensauffälligkeiten) festgestellt werden. Ihr aktuelles Befinden in der Schule variiert zum einen stärker von Situation zu Situation und ist zum anderen negativer ausgeprägt (Venetz et al., 2012).

Aber nicht nur situative Merkmale des Unterrichts, sondern auch Kontextmerkmale wie das soziale Klassenumfeld eines Jugendlichen hat einen Effekt auf das emotionale Erleben von Jugendlichen (Bellmore, Witkow, Graham \& Juvonen, 2004; Dishion \& Tipsord, 2011). Unklar ist jedoch, inwiefern das behaviorale Klassenumfeld im Sinne der Klassenkomposition in Bezug auf das Verhalten Zusammenhänge sozialer Interaktionen auf das emotionale Erleben vermittelt. Die Untersuchung von Effekten sozialer Interaktionen auf das emotionale Erleben von Jugendlichen in Abhängigkeit heterogener Verhaltensweisen und des behavioralen Klassenumfeldes bildet daher den Fokus dieses Beitrags.

\section{Heterogene Verhaltensweisen im Unterricht}

In zunehmend heterogenen Klassen sind herausfordernde Erlebens- und Verhaltensweisen bei Schülerinnen und Schülern sehr häufig zu finden (Bilz, 2008). Schülerinnen und Schüler mit Verhaltensauffälligkeiten haben einen erhöhten Bedarf an besonderer Aufmerksamkeit, da sie vielfach Konzentrations- und Aufmerksamkeitsschwierigkeiten und/oder ein erhöhtes Aggressions- oder Hyperaktivitätspotential aufweisen. Insbesondere Schülerinnen und Schüler mit deutlich erkennbaren - also nach außen hin sichtbaren - Erlebens- und Verhaltensauffälligkeiten bilden häufig den Fokus von Untersuchungen, da ihr Verhalten generell als "störender" wahrgenommen wird. Demgegenüber werden nach innen gerichtete Erlebens- und Verhaltensweisen aufgrund schwieriger zu diagnostizierender Symptome häufig übersehen. Darüber hinaus wurde lange Zeit angenommen, dass Kinder und Jugendliche weder unter Depressionen noch unter ernstzunehmenden Angstzuständen leiden können (Groen, Pössel \& Petermann, 2004). Dabei ist die Anzahl depressiver und ängstlicher Kinder in Deutschland in den vergangenen Jahren sogar gestiegen (Steffen, Akmatov, Holstiege \& Bätzing, 2018).

Der Bereich der Erlebens- und Verhaltensweisen lässt sich im Allgemeinen in die zwei diametralen Ausprägungstypen internalisierend oder externalisierend unterscheiden. Erstere beschreiben nach innen gerichtete Erlebens- und Verhaltensweisen, welche sich exemplarisch in depressiven und ängstlichen Symptomatiken äußern. Als externalisierend werden nach außen gerichtete Erlebens- und Verhaltensweisen bezeichnet, welche beispielsweise Aufmerksamkeitsprobleme, Hyperaktivität, Aggression sowie Delinquenz umfassen (Besser, Döhnert \& Stadelmann, 2019). Jeweilige Neigungen zu den gegenpoligen Ausprägungstypen verfügen somit über gegensätzliche Erscheinungsformen, was innerhalb des Schulkontexts zu unterschiedlichen Schwierigkeiten für die betroffenen Schülerinnen und Schüler als auch deren (schulisches) Umfeld führt. So erhalten Schülerinnen und Schüler mit vornehmlich externalisierenden Erlebens- und Verhaltensweisen häufig indirekte und direkte negative Rückmeldung vonseiten ihrer Mitschülerinnen und Mitschüler sowie von Lehrpersonen (Schulte-Köme, 2016). Disruptive Verhaltensweisen wie beispielsweise Hereinrufen im Unterricht oder fehlende Einhaltung der vereinbarten Spielregeln lassen sich als mögliche Gründe hierfür nennen. Im Gegensatz hierzu empfangen Schülerinnen und Schüler mit eher internalisierenden Erlebens- und Verhaltensweisen kaum Rückmeldungen vonseiten ihrer Mitschülerinnen und Mitschüler oder von Lehrpersonen - weder positiver noch negativer Art. Dies liegt insbesondere am inhärenten 
Charakteristikum zurückgezogener und unauffälliger Verhaltensweisen, sodass subklinische bzw. latente oder nicht direkt beobachtbare Ausprägungen internalisierender Erlebens- und Verhaltensweisen häufig übersehen werden (z.B. Lane, Oakes, Menzies \& Germer, 2014; Papandrea \& Winefield, 2011; Scherreiks \& Schwalbe, 2019).

Insbesondere das Jugendalter stellt eine kritische Phase für die Entwicklung und Manifestierung auffälligen Verhaltens und Erlebens dar, da diese Phase gekennzeichnet ist durch psychische, physische und auch soziale Veränderungen. Gleichzeitig nehmen freundschaftliche Beziehungen und die Bedeutung der Peers zu (Rubin, Bukowski \& Parker, 2006). Jugendliche mit internalisierendem oder externalisierendem Verhalten haben jedoch häufig Schwierigkeiten im zwischenmenschlichen und sozialen Bereich und werden oftmals aufgrund ihrer herausfordernden Verhaltensweisen - wie der sozialen Zurückgezogenheit oder der mangelnden Fähigkeit zur Affektregulation (Groen et al., 2004) - ausgegrenzt (Lüdeke, 2018). Die Neigung zu einer der beiden extremen Ausprägungsarten ist demnach mit exogen und endogen bedingten Problemverhaltensweisen der betroffenen Schülerinnen und Schüler verknüpft. Die wechselseitige Verstärkung des Problemverhaltens und der sozialen Umwelt führt zudem häufig in eine Art „Teufelskreis“ (Groen et al., 2004). Insofern überrascht es wenig, dass besonders während der Adoleszenz ein ausgeprägter $\mathrm{Zu}$ sammenhang zwischen Peerbeziehungen und internalisierendem (z.B. Bosacki, Dane, Marini \& YLC-CURA, 2007; La Greca \& Harrison, 2005; Prinstein, 2007) als auch externalisierendem Problemverhalten (z. B. Laird, Jordan, Dodge, Pettit \& Bates, 2001) besteht. So weisen beispielsweise die Ergebnisse von Bosacki und Mitarbeitenden (2007) auf einen mediierenden Effekt eines niedrigen Selbstwertes zwischen Peerbeziehungen und depressiven Episoden hin. Ein niedriger Selbstwert stellt sowohl ein typisches Charakteristikum internalisierender Erlebens- und Verhaltensweisen als auch ein störungsinhärentes Diagnosekriterium einer depressiven Episode dar. Hinsichtlich externalisierender Problemverhaltensweisen zeigen etwa Studienergebnisse der Arbeitsgruppe um Laird (2001), dass das Erleben repetitiver Ablehnung durch Peers die Entwicklung externalisierenden Problemverhaltens prädizieren.

Beeinträchtigungen in der Entstehung und Aufrechterhaltung sozialer Peerbeziehungen sind daher nicht selten (Lauer \& Renk, 2013; Olympia, Heathfield, Jenson, Majszak, Ramos-Matias \& Thacker, 2013). Vor diesem Hintergrund erscheint es umso bedeutender, in der Schule verschiedene Möglichkeiten zu sozialen Interaktionen mit Mitschülerinnen und Mitschülern zu gewährleisten (z. B. in Form von Gruppenarbeiten).

\section{Emotionales Erleben von Jugendlichen}

Das emotionale Erleben von Jugendlichen im Unterricht gilt nicht nur als wesentlicher Indikator des schulischen Wohlbefindens (z.B. Kullmann, Geist \& Lütje-Klose, 2015; Venetz, 2015), sondern kann zudem zur Beurteilung der Qualität sozialer Interaktionen herangezogen werden (Zurbriggen, 2018a). Wird während sozialer Interaktionen mit Peers ein positives situatives emotionales Erleben berichtet, so kann davon ausgegangen werden, dass diese Kontakte als besonders motivierend wahrgenommen werden. Im Gegensatz zum habituellen emotionalen Erleben bildet das situative emotionale Erleben den momentanen, aktuellen Erlebenszustand ab (z. B. Goetz, Preckel, Pekrun \& Hall, 2007) und variiert stärker von Situation zu Situation in Abhängigkeit von Kontextmerkmalen (z.B. Bolger \& Laurenceau, 2013).

Im vorliegenden Beitrag kann das situative emotionale Erleben als aktuelle Verarbeitung der subjektiv wahrgenommenen Emotionskomponenten beschrieben werden. Dabei umfassen diese affektive, kognitive, expressive, motivationale sowie physiologische Komponenten (z. B. Gerrig \& Zimbardo, 2008; Hascher \& Brandenberger, 2018; Izard, 1999; Reisenzein, 2018; Scherer, 2009). Zur Beschreibung des gesamten Spektrums des situativen affektiven Erlebens bietet sich das Circumplex-Modell emotionalen Erlebens nach Watson und Tellegen (1985; Watson, Wiese, Vaidya \& Tellegen, 1999) an, das einen dimensionalen Ansatz verfolgt. Im Gegensatz zu kategorialen Modellen, welche die Kategorisierung diskreter Emotionen in „Emotionsklassen“ (z. B. Freude oder Angst) sowie deren Entstehungsbedingungen und Funktionen theoriebasiert fokussieren, streben dimensionale Modelle an, „varianzstarke Faktoren zu identifizieren, anhand derer sich Emotionen faktorenanalytisch gruppieren lassen" (Pekrun \& Frenzel, 2009, S.688). Dies bietet u.a. den Vorteil die diffizile Abgrenzung von Emotionen zu Nicht-Emotionen weitestgehend unberührt zu lassen, woraus sich eine entsprechende Operationalisierbarkeit von emotionalem Erleben ergibt. Dimensionale Ansätze ermöglichen zudem eine Berücksichtigung von physiologischen oder motivationalen Emotionskomponenten (Schmidt-Atzert, 2009) und auch die Berücksichtigung neutraler bzw. weniger auffälliger Erlebenszustände. Dem Circumplex-Modell affektiven Erlebens liegen die beiden Dimensionen Valenz und Aktivierung zugrunde. Die Dimension der Valenz bezieht sich auf den aktuellen Zustand subjektiver Zufriedenheit und besitzt damit eine hedonistische Prägung (z. B. unzufrieden vs. zufrieden). Die Dimension der Aktivierung lässt sich wiederum in die beiden weite- 
ren Dimensionen der positiven Aktivierung (tief, z.B. gelangweilt vs. hoch, z. B. hoch interessiert) und der negativen Aktivierung (tief, z.B. entspannt vs. hoch, z.B. gestresst) unterteilen. Ist beispielsweise eine Schülerin positiv aktiviert, so ist sie voller Energie in der aktuellen Unterrichtssituation. Ist diese Schülerin tief positiv aktiviert, so ist sie in der aktuellen Situation eher gelangweilt oder desinteressiert.

\section{Bedeutung der Peers und des Klassenumfeldes für das emotionale Erleben im schulischen Kontext}

Peers sind bedeutsam im Leben von Jugendlichen - sowohl in der Freizeit, aber auch im schulischen Kontext. Im Rahmen der Selbstbestimmungstheorie wird auf die Relevanz des Zugehörigkeitsgefühls neben dem Bedürfnis nach Autonomie und nach Kompetenzerleben als wesentliches psychologisches Grundbedürfnis verwiesen (Ryan \& Deci, 2000). Der Theorie entsprechend erleben sich Schülerinnen und Schüler als selbstbestimmter und motivierter in ihrem Handeln, wenn sie sich ihrer Peer-Gruppe zugehörig und sich von ihr aufgenommen und unterstützt fühlen. Dies zeigt sich unter anderem auch in empirischen Befunden zum emotionalen Erleben bei Peerinteraktionen im Unterricht: So sind Schülerinnen und Schüler während Partner- oder Gruppenarbeiten motivierter und interessierter (hohe positive Aktivierung), gleichzeitig aber auch weniger gestresst oder nervös (niedrige negative Aktivierung) als in Einzelsituationen (z.B. Zurbriggen \& Venetz, 2018; Zurbriggen, Venetz \& Hinni, 2018. Dies wiederum schlägt sich auf das allgemeine positive emotionale Wohlbefinden nieder (z. B. Lyubomirsky, King \& Diener, 2005; Parker, Ciarrochi, Heaven, Marshall, Sahdra \& Kiuru, 2015).

Auch wenn die Bedeutsamkeit von Peers für das emotionale Erleben bereits vielfach bestätigt wurde, findet die Berücksichtigung der Peers auf Klassenebene - etwa im Sinne eines sozialen Klassenklimas - in der aktuellen Forschung (noch) wenig Aufmerksamkeit. Vereinzelt vorliegende Studienergebnisse geben Hinweise auf differente Zusammenhänge zwischen dem behavioralen Klassenumfeld und dem subjektiven emotionalen Erleben und Verhalten (Bellmore, Witkow, Graham \& Juvonen, 2004; Dishion \& Tipsord, 2011; Müller \& Zurbriggen, 2016). Gemäß der Studie von Mercer, McMillen und DeRosier (2009) verzeichnen Schülerinnen und Schüler in Klassenumfeldern mit einem durchschnittlich höheren Aggressionsniveau einen stärkeren Anstieg aggressiver Verhaltensund Erlebensweisen. Ein eher prosoziales Klassenklima dient wiederum als Prädiktor für einen Anstieg der Sozialkompetenzen der einzelnen Schülerinnen und Schüler (Hoglund \& Leadbeater, 2004). In einer weiteren Untersuchung zeigte sich, dass durch die jeweilige Klassenzusammensetzung beeinflussten sozialen und emotionalen Verhaltensnormen innerhalb einer Klassengemeinschaft als Bezugsnorm für das emotionale Erleben und Verhalten auf individueller Ebene, an denen sich die Jugendlichen orientieren, dienen können (Barth, Dunlap, Dane, Lochman \& Wells, 2004). Das behaviorale Klassenumfeld, das in den zitierten Studien über aggregierte Individualwerte der teilnehmenden Personen operationalisiert wurde, kann sich damit sowohl positiv als auch negativ auf das subjektive emotionale Erleben und Verhalten der einzelnen Schülerinnen und Schüler auswirken. Neben einem direkten Effekt auf sozial-emotionale Eigenschaften von Schülerinnen und Schüler kann das Klassenumfeld einen moderierenden Effekt auf den Zusammenhang etwa zwischen individuellen Faktoren (z. B. Aggressivität eines Jugendlichen) und sozial-emotionalen Outcomes (z.B. soziale Akzeptanz in der Klasse) haben (für einen Überblick Müller \& Zurbriggen, 2016). Theoretisch erklärt werden können moderierende Effekte des behavioralen Klassenumfeldes u. a. durch Kontrasteffekte: Zum Beispiel werden in weniger prosozialen Klassen soziale Kontakte mit Peers positiver bzw. stärker wahrgenommen (contrast effects, vgl. Sherif \& Hovland, 1961). Prosoziale Klassen lassen sich durch gegenseitigen Respekt oder Kooperationsbereitschaft charakterisieren. Ein solches Klima erleichtert Jugendlichen das Knüpfen und Aufrechterhalten von positiven Kontakten untereinander (Krawinkel, Südkamp, Lange \& Tröster, 2017). Ein soziales Miteinander ist in prosozialen Klassen vertrauter und fällt dadurch vermutlich weniger auf bzw. weniger emotional „ins Gewicht“.

\section{Ableitung der Fragestellungen}

Zusammenfassend ergeben sich zahlreiche Hinweise einer engen Verwobenheit zwischen emotionalen und sozialen Facetten im jugendlichen Erleben und Verhalten. Befunde aus vorangehenden Untersuchungen verweisen auf positive Effekte sozialer Interaktionen und sozialer Partizipation in der Klasse für das emotionale Erleben von Schülerinnen und Schüler mit einem sonderpädagogischen Unterstützungsbedarf (als offizieller Status) und mit Verhaltensauffälligkeiten (als klinisch auffälliger Gesamtproblemwert) der fünften und sechsten Jahrgangsstufe (Zurbriggen \& Venetz, 2016) sowie für Jugendliche mit einem sonderpädagogischen Unterstützungsbedarf der achten und neunten Jahrgangsstufe (Zurbriggen, Venetz \& Hinni, 2018). Um nicht - wie in Vorgängerstudien - nur Schülerinnen und 
Schüler mit diagnostizierten oder besonders auffälligen Verhaltensweisen zu berücksichtigen, sondern der gesamten Schülerschaft einer Klasse zu entsprechen, wird vor dem dargestellten Hintergrund der Bereich heterogener Verhaltensweisen aus einer dimensionalen, personzentrierten (statt kategorialen, variablenzentrierten) Perspektive betrachtet.

Fragestellung 1: Wie erleben Jugendliche mit heterogenen Verhaltensweisen soziale Interaktionen im Unterricht? Es wird angenommen, dass sich Schülerinnen und Schüler mit internalisierenden und externalisierenden Verhaltensweisen im emotionalen Erleben von sozialen Interaktionen mit Peers im Unterricht von ihren Mitschülerinnen und Mitschülern aufgrund der dargestellten Schwierigkeiten, Peerbeziehungen zu knüpfen und aufrechtzuerhalten, unterscheiden.

Trotz der Bedeutsamkeit von Peers für das emotionale Erleben - besonders während der Adoleszenz - existieren bislang kaum Forschungen zu Zusammenhängen des behavioralen Klassenumfeldes auf das emotionale Erleben von Jugendlichen unter Berücksichtigung ihrer individuellen heterogener Verhaltensweisen (siehe im Überblick Müller \& Zurbriggen, 2016). Daher ist neben den Effekten von Peerinteraktionen auf das emotionale Erleben von Jugendlichen der Effekt des behavioralen Klassenumfeldes - im Sinne der Klassenkomposition hinsichtlich des Verhaltens der Jugendlichen - von besonderem Interesse.

Fragestellung 2: Inwiefern moderiert das behaviorale Klassenumfeld Zusammenhänge zwischen sozialen Interaktionen und dem emotionalen Erleben? Es wird angenommen, dass der Effekt sozialer Interaktionen auf das emotionale Erleben in Abhängigkeit des behavioralen Klassenumfeldes variiert.

\section{Methode}

\section{Untersuchungsdesign und Erhebungsmethoden}

Die Datengrundlage dieses Beitrags basiert auf zwei Erhebungsmethoden: Zum einen wurden konventionelle schriftliche Fragebögen eingesetzt und zum anderen wurden Schülerinnen und Schüler mittels der experience sampling method (ESM; Hektner, Schmidt \& Csikszentmihalyi, 2007) befragt. Die ESM zählt zur Gruppe der intensive longitudinal methods (z. B. Bolger \& Laurenceau, 2013). Die Datenerhebung erfolgt dabei in situ ein- oder mehrmals täglich über eine oder auch mehrere Wochen hinweg.
Die zentrale Idee dabei ist „das komplexe Zusammenspiel von Erleben, Verhalten oder physiologischen Vorgängen von Personen und deren Lebensumwelt wiederholt und zeitnah zu untersuchen" (Venetz \& Zurbriggen, 2015, S.195). Dieser Ansatz bietet unter anderem den Vorteil, dass Merkmale aufgrund der zeitlichen Nähe zum Befragungsgegenstand mit einer hohen ökologischen Validität erfasst werden können. Auf diese Weise können zudem Retrospektionseffekte verringert werden (Stone \& LitcherKelly, 2006; Venetz \& Zurbriggen, 2016). Aufgrund wiederholter Messungen bietet die ESM darüber hinaus die Möglichkeit, Personen- und Situationsmerkmale miteinander zu verknüpfen. Sie ist zudem meist mit einem erhöhten Aufwand sowohl für die Untersuchungsteilnehmenden als auch für die Untersuchungsdurchführenden verbunden. Die recht komplexe Datenstruktur könnte ebenfalls als eine Herausforderung dieser Befragungsform ausgelegt werden.

In der vorliegenden Studie wurde die ESM während des Unterrichts im Laufe einer ganzen Schulwoche (montags bis freitags) eingesetzt. Vor Beginn der Untersuchung erhielten alle Schülerinnen und Schüler einer Klasse mehrere identische paper-and-pencil-Kurzfragebögen in BookletFormat, die sie während der Untersuchungswoche bei sich tragen sollten. Die Lehrpersonen wurden entweder mit einem Pager ausgestattet oder benutzten ihr Mobiltelefon für die Befragung. Zu insgesamt 14 zufällig generierten Zeitpunkten innerhalb der Untersuchungswoche (dementsprechend zwei bis drei Messzeitpunkte pro Schultag) wurde den Lehrpersonen ein Signal via Pager bzw. eine SMS via Mobiltelefon gesendet. Unmittelbar darauf wurden die Schülerinnen und Schüler gebeten, einen der kurzen ESM-Fragebögen auszufüllen, in dem unter anderem nach situativen Unterrichtsmerkmalen (z.B. Sozialform) und nach ihrem aktuellen emotionalen Erleben gefragt wurde. Das Ausfüllen eines Kurzfragebogens nahm zwei bis vier Minuten in Anspruch.

Am Ende der Untersuchungswoche wurde zusätzlich ein konventioneller schriftlicher Fragebogen eingesetzt, um demografische sowie habituelle Merkmale der Schülerinnen und Schüler zu erheben. Die Lehrpersonen gaben ebenfalls mittels schriftlicher Fragebögen weitere Auskunft über ihre Schülerinnen und Schüler. Die Datenerhebungen fanden im zweiten Schulhalbjahr 2008/2009 statt ${ }^{1}$.

\section{Stichprobe}

Insgesamt nahmen $N=719$ Schülerinnen und Schüler aus 40 inklusiven Schulklassen an der Untersuchung teil, die 
im Rahmen eines größer angelegten schweizerischen Forschungsprojekts gewonnen werden konnten (Venetz et al., 2012). Davon besuchte der Großteil die sechste Jahrgangsstufe ( $n=660,91.8 \%), 7.6 \%$ der Stichprobe stammten aus der fünften Jahrgangsstufe. Diese Verteilung lässt sich dadurch erklären, als dass sieben der 40 Klassen jahrgangsübergreifend unterrichtet wurden. $49 \%$ der Teilnehmenden $(n=352)$ waren weiblich; bei 151 Schülerinnen und Schülern (21.5\%) ist Deutsch die Zweitsprache. Das durchschnittliche Alter betrug zum Zeitpunkt der Befragung $M=$ 12.2 Jahre $(S D=0.8)$.

Im Laufe einer Untersuchungswoche füllten die Schülerinnen und Schüler insgesamt 8870 ESM-Fragebögen aus. Demnach liegen durchschnittlich $M=12.3$ Kurzfragebögen pro Schüler bzw. Schülerin vor. Somit liegt in dieser Studie eine genestete Datenstruktur vor, in der Messzeitpunkte in Schülerinnen und Schüler und diese wiederum in Klassen geschachtelt sind.

\section{Messinstrumente}

Das aktuelle emotionale Erleben der Schülerinnen und Schüler wurde innerhalb der ESM-Kurzfragebögen erfasst. Hierzu wurden die PANAVA-Kurzskalen (Schallberger, 2005) verwendet, die auf dem Circumplex-Modell affektiver Zustände (Watson \& Tellegen, 1985) basieren. Diesem Modell zufolge kann das emotionale Erleben anhand zweier Dimensionen - der positiven Aktivierung (PA) und der negativen Aktivierung (NA) - beschrieben werden. Die beiden Dimensionen werden mit jeweils vier Gegensatzpaaren operationalisiert (,Wie fühltest du dich kurz vor dem Signal?" - z.B. „lustlos vs. hoch motiviert" für PA, z.B. „gestresst vs. entspannt" für NA). Die Antworten werden anhand einer 7-stufigen bipolaren Likertskala, deren Endpole durch die Gegensatzpaare beschrieben werden, gemessen. Die Reliabilität $(\omega)$ der Skalen beträgt .71 (PA) bzw. .69 (NA) auf Situationsebene und .92 (PA) bzw. .88 (NA) auf Personebene.

Die Peerinterkationen wurden ebenfalls innerhalb der ESM-Fragebögen erfasst. Konkret wurden die Schülerinnen und Schüler mehrmals täglich gebeten, Auskunft zum aktuellen sozialen Kontext im Unterricht anhand eines einzelnen Items zu geben („Wie bist du im Moment beschäftigt?" mit den Antwortkategorien „allein“, „zu zweit“, „in der Gruppe“, „mit Klasse“, „höre zu-Lehrperson“, „höre zu-Mitschüler/Mitschülerin"). Auf der Grundlage dieser Information wurde die Dummy-Variable Peerinteraktion gebildet, bei der die Ausprägung 0 für nicht stattfindende Peerinteraktion (Antwortkategorien „allein“, „höre zu [Lehrperson oder Mitschüler / Mitschülerin] ") und die Ausprägung 1 für eine stattfindende Peerinteraktionen (Antwortkategorien: „zu zweit“, „in der Gruppe“, „mit Klasse“) steht.
Zur Erhebung von Verhaltensweisen füllten die Klassenlehrpersonen die deutsche Version für Lehrerinnen und Lehrer des Strengths and Difficulties Questionnaire's (SDQ; Goodman, 1997) für jeden Schüler bzw. jede Schülerin der Klasse aus. Mit jeweils fünf Items pro Skala werden die vier Konstrukte „Verhaltensprobleme“ (z.B. „Er/Sie streitet sich oft mit anderen Kindern oder schikaniert sie.“), „Hyperaktivität“ (z.B. „Er/Sie ist leicht ablenkbar, unkonzentriert."), „emotionale Probleme“ (z.B. „Er/Sie ist oft unglücklich oder niedergeschlagen; weint häufig.") und „Peerprobleme“ (z.B. „Er/Sie wird von anderen gehänselt oder schikaniert.“; Skalen zum Problemverhalten) sowie das Konstrukt „prosoziales Verhalten“ (z.B. „Er/Sie ist hilfsbereit, wenn andere verletzt, krank oder betrübt sind.“) gemessen. Die Einschätzungen werden auf einer dreistufigen Skala vorgenommen $(0=$ nicht zutreffend, 1 = teilweise zutreffend, 2 = eindeutig zutreffend). Die Reliabilitäten der fünf Subskalen sind mit $.70 \leq \omega \leq .87$ in einem angemessenen Bereich.

Die Variable „behaviorale Klassenkomposition“ wurde basierend auf den SDQ-Skalen gebildet. Für jeden Schüler bzw. jede Schülerin wurden jeweils zwei individuelle Umgebungswerte in Bezug auf sein bzw. ihr Klassenumfeld hinsichtlich des Problemverhaltens und des prosozialen Verhaltens ermittelt, die durch die entsprechenden SDQKlassenmittelwerte abzüglich des individuellen Werts berechnet wurden, um dadurch die Konfundierung von Individual- und Klasseneffekt zu kontrollieren (Hox, 2010; Müller \& Zurbriggen, 2016). Die deskriptiven Statistiken und die Korrelationen der Subskalen auf Individual- und Klassenebene sind in Tabelle $1 \mathrm{zu}$ finden. Grundsätzlich zeigen sich die erwarteten Zusammenhänge der untersuchten Variablen.

\section{Datenanalysen}

Zur Identifizierung von relativ homogenen Subgruppen hinsichtlich der Verhaltenskompetenzen innerhalb der Schülerschaft wurde eine Reihe von latenten Profilanalysen (LPA) durchgeführt. Bei dieser personzentrierten Herangehensweise werden Typologien ermittelt, in der die Personen (bzw. Schülerinnen und Schüler) auf Grundlage ihrer spezifischen Kombination von Stärken und Schwächen (im Bereich Verhalten) in qualitativ und quantitativ unterschiedliche Profile eingeteilt werden (Morin \& Marsh, 2015). Die Klassifizierung der latenten Profile erfolgte basierend auf den Testwerten der vier SDQ-Problemskalen. Zur Bestimmung der optimalen Anzahl Profile wurden zum einen die folgenden drei Informationskriterien herangezogen: Akaikes Informationskriterium (AIC), Bayessches Informationskriterium (BIC) und stichprobengrößenadjustiertes BIC (SA-BIC). Grundsätzlich ist jeweils 
Tabelle 1. Deskriptive Statistiken und Korrelationen des Strength and Difficulties Questionnaires auf Individual- und Klassenebene

\begin{tabular}{|c|c|c|c|c|c|c|c|c|}
\hline Individualabene & $M^{\mathrm{a}}$ & $S D$ & (2) & (3) & (4) & (5) & (6) & (7) \\
\hline (1) Verhaltensprobleme & 1.28 & 1.87 & $.62^{\star}$ & $.16^{\star}$ & $.23 *$ & $-.58 *$ & $.32 *$ & $-.16 *$ \\
\hline (2) Hyperaktivität & 2.88 & 2.86 & & $.25^{\star}$ & $.17^{\star}$ & $-.46^{\star}$ & $.31 *$ & $-.14^{*}$ \\
\hline (3) emotionale Probleme & 1.64 & 1.93 & & & $.43 *$ &.-.05 & $.30 *$ & -.04 \\
\hline (4) Peerprobleme & 1.52 & 1.88 & & & & $-.30 \star$ & $.33 *$ & $-.20 *$ \\
\hline (5) prosoziales Verhalten & 7.40 & 2.48 & & & & & $-.22^{\star}$ & $.51 *$ \\
\hline \multicolumn{9}{|l|}{ Klassenebene } \\
\hline (6) Problemverhalten & 1.83 & 0.67 & & & & & & $-.43 *$ \\
\hline (7) prosoziales Verhalten & 7.40 & 1.26 & & & & & & \\
\hline
\end{tabular}

Anmerkungen: $N$ $=719, N_{K}$ = 40. ${ }^{\mathrm{a}}$ range $0-10 .{ }^{\star} p<.05$.

das Modell mit dem kleinsten Wert zu favorisieren. Zum anderen dienten für die Modellauswahl der Vuong-LoMendell-Rubin-Test (VLMRT), der Lo-Mendell-Rubin likelihood ratio test (LMR-LRT; Lo, Mendell \& Rubin, 2001) sowie der bootstrap likelihood ratio test (BLRT). Bei diesen Log-likelihood-Differenztests wird ein Modell hinsichtlich einer signifikanten Veränderung zum vorangehenden Modell (mit einer Klasse weniger) getestet. Ist die Differenz signifikant, wird das Modell mit einer Klasse mehr präferiert. Hinweise für die Güte eines Modells bieten zudem die Entropie sowie die mittlere Klassenzuordnungswahrscheinlichkeit. Eine hohe Entropie kann als Reliabilität der Klassifikation der Personen zu den latenten Klassen interpretiert werden. Nicht zuletzt sind bei der Auswahl der besten Klassenlösung inhaltliche bzw. theoretische Überlegungen sowie die Sparsamkeit zu berücksichtigen.

Die Fragen nach Unterschieden des emotionalen Erlebens bei Peerinteraktionen im Unterricht je nach Profiltyp wurden anhand von Multilevel-Strukturgleichungsmodellen unter Verwendung eines robusten Maximum-Likelihood-Schätzers (MLR) untersucht. Auf Level 1 der ZweiEbenen-Modelle wurden die Zeitpunkte bzw. Situationen spezifiziert, auf Level 2 die Personen bzw. Schülerinnen und Schüler. Die Intraklassenkorrelationen (ICC) auf Personenebene lagen für die acht PANA-Items zwischen .23 und .36, d.h. zwischen 23 und $36 \%$ der Gesamtvarianz ist auf Unterschiede zwischen Schülerinnen und Schüler zurückzuführen. Da mit ICCs zwischen .02 und .05 kaum klassenbedingte Varianz in den PANA-Items vorhanden war, wurde keine dritte Ebene modelliert.

Die Untersuchung des emotionalen Erlebens in Abhängigkeit des Profiltyps setzt die Äquivalenz der Messungen in den PANA-Items voraus. Deshalb wurde in einem ersten Schritt das Messmodell im Rahmen von multiplen Multilevel-Gruppenvergleichen auf Messinvarianz überprüft. In einem zweiten Schritt erfolgte die Überprüfung des Strukturmodells von PANA auf Gleichheit der Varianzen, Kovarianzen und Mittelwerte, um Aufschluss über die Struktur des emotionalen Erlebens je nach Profiltyp zu erhalten. Zur Beurteilung der Güte der Modellpassung wurden der inferenzstatistische MLR- $\chi^{2}$-Test sowie die folgenden drei approximativen Fit-Indizes herangezogen: Comparative Fit Index (CFI), Root Mean Square Error of Approximation (RMSEA) und Standardized Root Mean Residual (SRMR). Gemäß den Richtwerten von Hu und Bentler (1999) sprechen ein CFI $\geq .95$, ein RMSEA $\leq 0.06$ und ein SRMR $\leq .08$ für eine gute Modellpassung. Für den Modellvergleich wurde neben dem MLR- $\chi^{2}$-Differenztest die CFI-Differenz berücksichtigt, wobei eine CFI-Differenz $\geq .01$ als bedeutsam zu werten ist (Chen, 2007).

Schließlich wurden die Effekte von Peerinteraktion (Level-1-Prädiktor) und der Klassenkomposition bezüglich Problemverhalten und prosozialem Verhalten (Level-2Prädiktoren) auf das emotionale Erleben im Rahmen von Multilevel-Strukturgleichungsmodellen untersucht. Dazu wurden intercepts-and-slopes-as-outcomes-Modelle mit zufällig variierenden Regressionsgewichte (random slopes) für den Zusammenhang von Peerinteraktion und emotionalem Erleben und mit cross-level-Interaktionen zwischen den random slopes und den beiden Klassenkompositionsvariablen spezifiziert.

Alle Analysen erfolgten mit Mplus Version 8.0 (Muthén \& Muthén, 1998-2017).

\section{Ergebnisse}

\section{Latente Profiltypen}

Für die Identifizierung von relativ homogenen Subgruppen innerhalb der Schülerschaft wurden LPAs bis zu fünf Klassen durchgeführt. Wie Tabelle 2 zu entnehmen ist, verringern sich die Werte der Informationskriterien AIC, BIC und SA-BIC mit zunehmender Anzahl latenter Klassen. Die Entropie liegt bei allen Modellen bei mindestens 
Tabelle 2. Fitindizes der latenten Profilanalysen (LPA)

\begin{tabular}{lcccccccc}
\hline Latente Klassen & Log-likelinood & AIC & BIC & SA-BIC & VLMRT & LMR-LRT & BLRT & Entropie \\
\hline 1 & -6195.27 & 12406.53 & 12443.12 & 12417.72 & - & - & $<$ \\
2 & -5890.73 & 11807.46 & 11866.93 & 11825.64 & $<.001$ & $<.001$ & $<.001$ & 0.93 \\
3 & -5768.85 & 11573.69 & 11656.02 & 11598.86 & .003 & .004 & $<.001$ & 0.90 \\
4 & -5647.06 & 11340.12 & 11445.32 & 11372.29 & .004 & .004 & $<.001$ & 0.91 \\
5 & -5588.39 & 11232.79 & 11360.85 & 11271.94 & .091 & .096 & $<.001$ & 0.92 \\
\hline
\end{tabular}

Anmerkungen: $N=719$. AIC = Akaikes Informationskriterium; $\mathrm{BIC}=$ Bayessches Informationskriterium; $\mathrm{SA}-\mathrm{BIC}=$ stichprobengrößenadjustiertes BIC; $\mathrm{VLMRT}=$ $p$-Wert des Vuong-Lo-Mendell-Rubin-Tests; LMR-LRT = p-Wert des Lo-Mendell-Rubin-Likelihood-Ratio-Tests; BLRT = p-Wert des Bootstrap-LikelihoodRatio-Differenzen-Tests.

.90, mit .93 am höchsten für die Zwei-Klassen-Lösung. Gemäß dem VLMRT und dem LMR-LRT passen die DreiKlassen- und die Vier-Klassen-Lösung jedoch besser zu den Daten als das jeweils sparsamere Modell. Der BLRT legt jeweils das Modell mit der nächsthöheren Anzahl Klassen nahe.

Die nähere Betrachtung der Mittelwerte in den vier SDQ-Problemskalen zeigte, dass die Drei- und die VierKlassen-Lösung mit einer Ausnahme sehr ähnliche Profiltypen ergaben: Ein Profiltyp unterteilte sich bei der Vier-Klassen-Lösung in zwei Subgruppen mit tieferen vs. höheren Ausprägungen in allen Skalen, wobei die letztere nur knapp $4.6 \%$ der Schülerschaft umfasste. Deshalb und aus inhaltlichen Erwägungen wurde schließlich die DreiKlassen-Lösung als finales Modell gewählt. Die mittlere
Klassenzuordnungswahrscheinlichkeit mit Werten zwischen .90 und .97 unterstützte die Entscheidung.

In Abbildung 1 sind die Mittelwerte der SDQ-Problemskalenwerte zu den drei Profiltypen der Drei-KlassenLösung dargestellt. Die weitaus größte Anzahl der Schülerinnen und Schüler wurden dem ersten Profiltyp mit durchwegs tiefen Werten zugeordnet. Bei diesen rund $74 \%(n=527)$ wurde ein unauffälliges bzw. adaptives Verhalten beobachten. Knapp 12\% $(n=85)$ ließen sich einem Profiltyp zuordnen, der in erster Linie durch sehr hohe Werte bezüglich emotionaler Probleme beschrieben werden kann. Da dies ein zentrales Merkmal von internalisierenden Erlebens- und Verhaltensweisen bildet, wird der zweite Profiltyp entsprechend benannt. Etwa 15\% $(n=$ 104) der Schülerinnen und Schüler wurden dem dritten

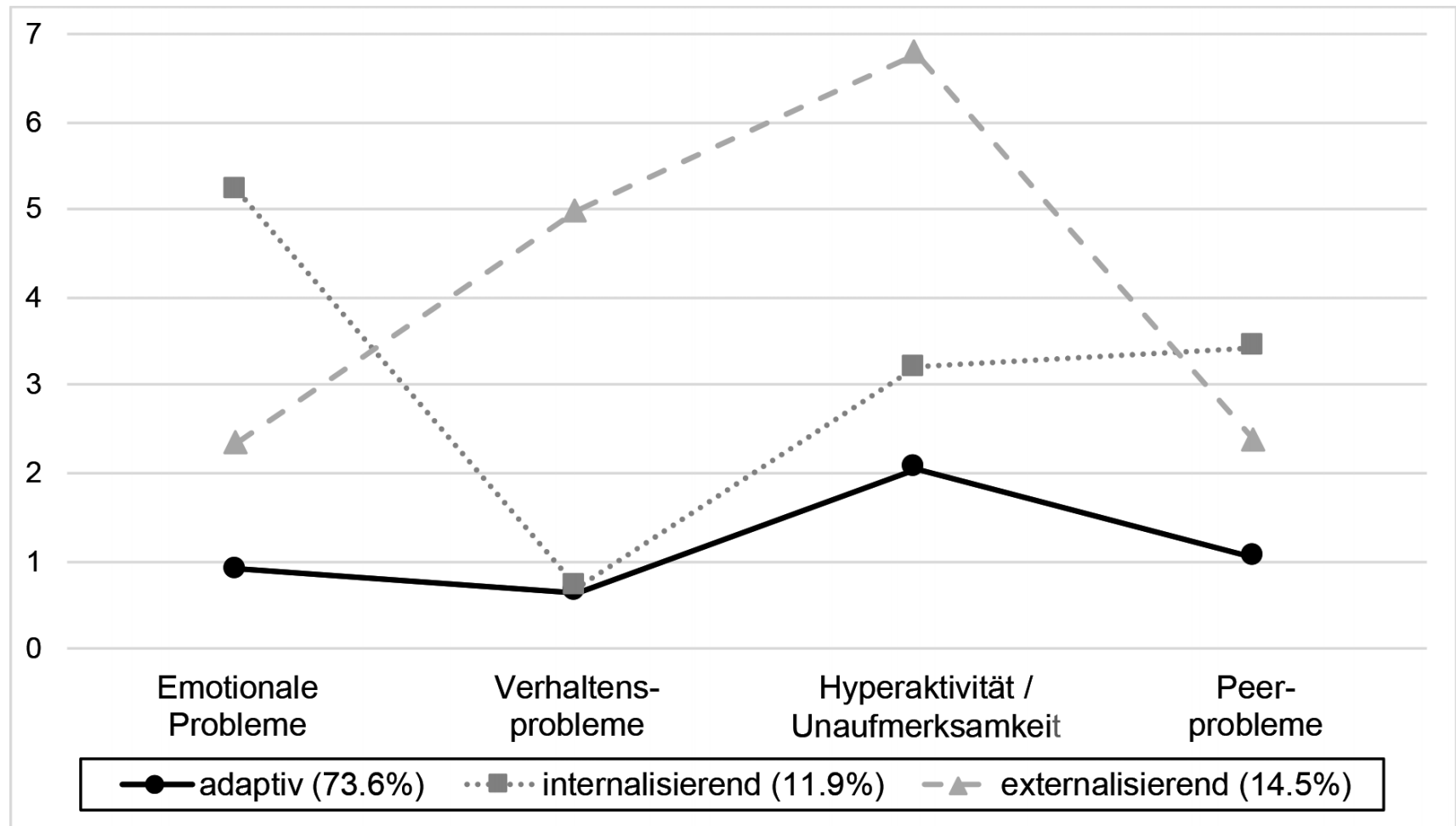

Abbildung 1. Mittelwerte der SDQ-Problemskalenwerte der drei Profiltypen. 
Profiltypen zugeteilt, der sich durch ausgeprägte Verhaltensprobleme und auffallend hohe Hyperaktivität und damit durch externalisierende Verhaltensweisen charakterisieren lässt. In diesem Profiltyp sind Jungen (22.3\%) überproportional häufiger vertreten als Mädchen (6.5\%). Während beim internalisierenden Profiltyp die Anteile ausgewogen sind (Jungen: 10.7\%; Mädchen: 11.9\%), sind beim adaptiven Profiltyp die Mädchen (80.4\%) stärker vertreten als die Jungen (67.0\%). Zwischen Geschlecht und Profiltyp besteht ein signifikanter Zusammenhang $\left(\chi^{2}\right.$ $(1, N=716)=35.62, p<.001)$, der jedoch eher klein ist (Cramer's V $=.22$ ).

Bei der Validierung der Drei-Klassen-Lösung anhand der Selbstbeschreibungen der Persönlichkeit zeigten sich bei einigen Dimensionen signifikante, teilweise recht große Unterschiede zwischen den Profiltypen. So beschrieben sich Schülerinnen und Schüler mit internalisierendem Verhalten als weniger emotional stabil $(\Delta M=-0.40)$ und weniger extravertiert bzw. zurückhaltender $(\Delta M=-0.51)$ als ihre Peers mit adaptivem Verhalten. Schülerinnen und Schüler mit externalisierendem Verhalten wiederum gaben an, weniger gewissenhaft $(\Delta M=-0.41)$ und deutlich weniger verträglich bzw. streitsüchtiger $(\Delta M=-0.69)$ als ihre Peers mit adaptivem Verhalten zu sein.

\section{Struktur emotionalen Erlebens im Unterricht}

Vor der Untersuchung der Struktur des emotionalen Erlebens in Abhängigkeit des Profiltypen galt es die PANA-
Items auf Messinvarianz zu prüfen. Wie Tabelle 3 zu entnehmen ist, wies das konfigurale Modell (M1) einen guten Modellfit auf. Das metrische Modell (M2) passte nicht signifikant schlechter auf die Daten als das konfigurale Modell $\left(\Delta M L R \chi^{2}=21.66, d f=32, p=.916\right)$. Das skalare Modell (M3) schnitt im Vergleich zum vorangehenden ebenfalls nicht signifikant schlechter ab $(\Delta \operatorname{MLR} \chi 2=19.00, d f=12$, $p=$.089). Darüber hinaus war bei beiden Vergleichen die CFI-Differenz geringfügig, sodass bei den PANA-Items von skalarer Messinvarianz ausgegangen werden kann.

Anschließend wurde im Rahmen von weiteren multiplen Gruppenvergleichen die Varianzen (M4), die Kovarianzen (M5) und die Mittelwerte (M6) überprüft, indem diese sukzessive über die Gruppen bzw. Profiltypen restringiert wurden. Um jeweils eine nicht-signifikant schlechtere Modellpassung im Vergleich zum skalaren Modell (M3) zu erreichen, mussten zwei Varianzen (M4a), eine Kovarianz (M5a) und zwei Mittelwerte (M6a) frei geschätzt werden. Das finale Modell (M6a) mit partieller Messinvarianz des Strukturmodells von PANA wies einen guten Fit mit den Daten auf $\left(\mathrm{MLR}^{2}=442.98, \mathrm{CFI}=.970\right.$, RMSEA $=.029$, $\left.\mathrm{SRMR}_{\mathrm{W} / \mathrm{B}}=.033 / .074\right)$.

In Tabelle 4 sind die Kennwerte des finalen Modells (M6a) nach Profiltyp aufgeführt. Anhand der Varianzanteile von PA und NA auf Level 2 und Level 1 lässt sich schließen, dass das emotionale Erleben im Unterricht im Allgemeinen stärker von der Situation (ca. 60\%) als von der Person (ca. $40 \%$ ) bestimmt wird. Die Struktur des emotionalen Erlebens ist bei Schülerinnen und Schüler mit internalisierendem Verhalten vergleichbar zu jenen mit adaptivem Verhalten. Unterschiede zeigen sich einzig

Tabelle 3. Mess- und Strukturmodelle für PANA: Fit-Statistiken der multiplen Gruppenvergleiche

\begin{tabular}{|c|c|c|c|c|c|c|c|c|c|c|c|}
\hline \multicolumn{2}{|c|}{ Modell } & $d f$ & MLR- $\chi^{2}$ & $\mathrm{CFI}$ & RMSEA & $\mathrm{SRMR}_{\mathrm{W} / \mathrm{B}}$ & Vergleich & $\Delta M L R-\chi^{2}$ & $\Delta d f$ & $p$ & $\Delta \mathrm{CFI}$ \\
\hline \multicolumn{12}{|c|}{ Überprüfung des Messmodells } \\
\hline M1 & Konfigurales Modell & 103 & $364.28 *$ & .972 & .029 & $.030 / .062$ & & & & & \\
\hline M2 & Metrisches Modell & 135 & $414.65^{\star}$ & .970 & .027 & $.031 / .069$ & M1 vs. M2 & 21.66 & 32 & .916 & -.002 \\
\hline M3 & Skalares Modell & 147 & $439.26 *$ & .969 & .026 & $.031 / .068$ & M3 vs. M2 & 19.00 & 12 & .089 & -.001 \\
\hline \multicolumn{12}{|c|}{ Überprüfung des Strukturmodells } \\
\hline M4 & M3 + Faktorvarianzen gleich & 155 & $455.03^{\star}$ & .968 & .026 & $.040 / .073$ & M4 vs. M3 & 17.96 & 8 & .022 & -.001 \\
\hline M4a & M4, 2 Varianzen frei geschätzt & 153 & $440.65^{\star}$ & .969 & .025 & $.032 / .073$ & M4a vs. M3 & 3.87 & 6 & .694 & .000 \\
\hline M5 & M4a + Faktorenkovarianzen gleich & 157 & $456.64^{*}$ & .968 & .025 & $.043 / .074$ & M5 vs. M3 & 20.11 & 10 & .028 & -.001 \\
\hline M5a & M5, 1 Kovarianz frei & 156 & 439.91* & .970 & .025 & $.033 / .074$ & M5a vs. M3 & 4.79 & 9 & .852 & .001 \\
\hline M6 & M5a + Faktorenmittelwerte gleich & 160 & $458.89 *$ & .968 & .025 & $.033 / .075$ & M6 vs. M3 & 19.94 & 13 & .097 & -.001 \\
\hline M6a & M6, 2 Mittelwerte frei & 158 & $442.98 *$ & .970 & .029 & $.033 / .074$ & M6avs. M3 & 6.08 & 11 & .868 & -.000 \\
\hline \multicolumn{12}{|c|}{ Überprüfung der Regressionskoeffizienten } \\
\hline M7 & Alle Regressionskoeffizienten frei & 212 & $572.99 *$ & .967 & .030 & $.033 / .068$ & & & & & \\
\hline M8 & Alle Regressionskoeffizienten gleich & 224 & $587.65^{*}$ & .966 & .024 & $.033 / .078$ & M8 vs. M7 & 8.46 & 12 & .749 & -.001 \\
\hline
\end{tabular}

Anmerkungen: $N_{\text {Level } 1}=8835, N_{\text {Level } 2}=719 . \mathrm{CFI}=$ Comparative Fit Index, RMSEA = Root Mean Square Error of Approximation, SRMR = Standardized Root Mean Square Residual (SRMR $=$ within, $S R M R_{B}=$ between); $\Delta M L R-\chi^{2}=M L R-\chi^{2}$-Differenz (robuster Maximum-Likelihood-Schätzer). ${ }^{*} p<.001$. 
Tabelle 4. Struktur des emotionalen Erlebens im Unterricht nach Profiltyp (Modell 6a)

\begin{tabular}{lccc}
\hline & Adaptiv & Internalisierend & Externalisierend \\
\hline Zeitpunktebene (Level 1) & & & 1.59 \\
Varianz PA & 1.19 & 1.19 & 1.34 \\
Varianz NA & 1.00 & 1.00 & $-1.10(-.76)$ \\
Kovarianz (Korrelation) & $-0.74(-.68)$ & $-0.74(-.68)$ & 0.87 \\
\hline Personebene (Level 2) & & & 0.87 \\
Varianz PA & 0.87 & 0.67 & $-0.59(-.77)$ \\
Varianz NA & 0.67 & $-0.59(-.77)$ & -0.24 \\
Kovarianz (Korrelation) & $-0.59(-.77)$ & 0.00 & 0.45 \\
Mittelwert PA & 0.00 & 0.00 & \\
Mittelwert NA & 0.00 & & \\
\hline
\end{tabular}

Anmerkungen: $N_{\text {Level 1 }}=8809, N_{\text {Level 2 }}=719$. Frei geschätzte Parameter sind fett markiert.

Alle Parameter ungleich 0 sind signifikant $(p<.05)$.

beim dritten Profiltyp: Bei Schülerinnen und Schülern mit externalisierenden Verhaltensweisen sind PA und NA noch etwas deutlicher von der Situation geprägt (ca. 65\%). Sie zeigen eine höhere intraindividuelle Variabilität im emotionalen Erleben, d.h. PA und NA variieren stärker über die verschiedenen Unterrichtssituationen hinweg. Auf deskriptiver Ebene (ohne Berücksichtigung der Mehrebenenstruktur) lassen sich ähnliche Mittelwerte der positiven Aktivierung in den drei Gruppen erkennen, die negative Aktivierung der Schülerinnen und Schüler des externalisiernenden Profiltyps fällt vergleichsweise höher aus (adaptiver Profiltyp - PA: $M=4.78, S D=1.43$, NA: $M=$ 2.65, $S D=1.30$; internalisierender Profiltyp - PA: $M=4.66$, $S D=1.33$, NA: $M=2.79, S D=1.29$; externalisiernder Profiltyp - PA: $M=4.56, S D=1.53$, NA: $M=3.11, S D=1.41)$. Die Korrelationen zwischen PA und NA sind bei allen drei Profiltypen recht hoch. Beim externalisierenden Profiltyp korrelieren PA und NA auf Zeitpunktebene etwas höher als bei den anderen Profiltypen. Wie die Mittelwerte zeigen, erleben Schülerinnen und Schüler dieses Profiltyps zudem im Unterricht im Durchschnitt weniger PA und mehr NA als ihre Peers.

\section{Effekte von Peerinteraktion und Klassenkomposition auf das emotionale Erleben}

Zur Beantwortung der Fragestellungen wurden die Prädiktoren Peerinteraktion (Level 1), Klassenkomposition bezüglich Problemverhalten und prosozialem Verhalten (Level 2) sowie die cross-level-Interaktionen in das Multilevel-Strukturgleichungsmodell eingeführt (M7, ModellfitStatistiken in Tabelle 3). Zuerst wurde die Äquivalenz der Regressionskoeffizienten über die Gruppen hinweg ge- testet (M8). Aufgrund des nicht signifikanten MLR- $\chi^{2}$ Differenztests und der geringen CFI-Differenz kann davon ausgegangen werden, dass diese Parameter über die drei Profiltypen hinweg gleich funktionieren.

Die Ergebnisse zum Level-1-Prädiktor zeigten, dass das gemeinsame Arbeiten mit Peers im Unterricht generell einen positiven Effekt auf das emotionale Erleben von Schülerinnen und Schülern hat - und dies im doppelten Sinne: Zum einen sind sie engagierter und motivierter $(\mathrm{PA}+)$ und zum anderen weniger gestresst oder verärgert (NA-). Wie in Tabelle 5 ersichtlich ist, haben Peerinteraktionen einen stärkeren Effekt auf das emotionale Erleben von Schülerinnen und Schülern mit internalisierenden $(\triangle \mathrm{PA}=.21$; $\triangle \mathrm{NA}=-.24)$ oder mit externalisierenden Verhalten $(\triangle \mathrm{PA}=$ $.37 ; \Delta \mathrm{NA}=-.23$; jeweils $p<.05)$. Im Detail steigt der Skalenwert auf der 7-stufigen Skala der positiven Aktivierung während Peerinteraktionen also um .21 (bzw. um .24) bei Schülerinnen und Schülern mit internalisierendem (bzw. externalisierendem) Verhalten im Vergleich zu ihren Peers mit adaptivem Verhalten.

Beim externalisierenden Profiltyp ist der Effekt auf PA somit noch ausgeprägter als beim internalisierenden Profiltyp. Allerdings waren bei dem externalisierenden Profiltyp bereits höhere Durchschnittswerte von PA und NA zu verzeichnen.

Hinsichtlich der Klassenkomposition bezüglich Problemverhalten und prosozialem Verhalten, die wie zu erwarten negativ korrelieren (siehe Tabelle 1), ergab sich kein moderierender Effekt auf den Zusammenhang zwischen Peerinteraktion und emotionalem Erleben im Unterricht. Bei der Klassenkomposition bezüglich prosozialem Verhalten zeigte sich ein geringfügiger negativer Effekt auf PA für die ersten beiden Profiltypen. Für Schülerinnen und Schüler mit adaptivem oder mit internalisierendem Verhalten scheint der positive Effekt des gemein- 
Tabelle 5. Effekte von Peerinteraktion und Klassenkomposition bezüglich Problemverhalten und prosozialem Verhalten auf das emotionale Erleben im Unterricht

\begin{tabular}{|c|c|c|c|c|c|c|}
\hline & \multicolumn{2}{|c|}{ Adaptiv } & \multicolumn{2}{|c|}{ Internalisierend } & \multicolumn{2}{|c|}{ Externalisierend } \\
\hline & Estimate & SE & Estimate & $S E$ & Estimate & $S E$ \\
\hline \multicolumn{7}{|c|}{ Positive Aktivierung (PA) } \\
\hline Intercept $\left(\gamma_{00}\right)$ & $0.00^{a}$ & .00 & -0.11 & .11 & -0.24 & .11 \\
\hline Peerinteraktion $\left(\mathrm{y}_{10}\right)$ & $0.00^{a}$ & .00 & 0.21 & .09 & 0.37 & .09 \\
\hline$P V\left(\gamma_{01}\right)$ & 0.03 & .02 & 0.00 & .04 & 0.06 & .03 \\
\hline $\mathrm{SOZ}\left(\mathrm{y}_{02}\right)$ & 0.07 & .04 & 0.08 & .08 & 0.12 & .11 \\
\hline$P V *$ peerlnt $\left(V_{11}\right)$ & -0.01 & .02 & 0.00 & .03 & 0.04 & .04 \\
\hline SOZ* peerlnt $\left(\mathrm{V}_{21}\right)$ & -0.09 & .03 & -0.13 & .06 & 0.12 & .11 \\
\hline \multicolumn{7}{|c|}{ Negative Aktivierung (NA) } \\
\hline Intercept $\left(\mathrm{y}_{00}\right)$ & $0.00^{\mathrm{a}}$ & .00 & 0.11 & .11 & 0.45 & .10 \\
\hline Peerinteraktion $\left(\mathrm{y}_{10}\right)$ & $0.00^{a}$ & .00 & -0.24 & .10 & -0.23 & .10 \\
\hline$P V\left(\gamma_{01}\right)$ & 0.01 & .02 & -0.01 & .04 & -0.04 & .03 \\
\hline $\mathrm{SOZ}\left(\mathrm{\gamma}_{02}\right)$ & -0.02 & .03 & 0.08 & .08 & -0.08 & .08 \\
\hline$P V *$ peerlnt $\left(Y_{11}\right)$ & 0.00 & .02 & -0.01 & .04 & -0.02 & .04 \\
\hline SOZ* peerlnt $\left(V_{21}\right)$ & 0.02 & .03 & -0.06 & .08 & -0.05 & .12 \\
\hline
\end{tabular}

Anmerkungen: PV = Klassenkomposition Problemverhalten; SOZ = Klassenkomposition prosoziales Verhalten; peerlnt = Peerinteraktion. Signifikante Parameter $(p<.05)$ sind fett markiert. ${ }^{a}$ Zwecks Modellidentifikation auf 0 gesetzt.

samen Arbeitens mit Peers in verhältnismäßig prosozialen Klassen etwas weniger stark ausgeprägt zu sein als in weniger prosozialen Klassen. Dieser moderierende Effekt ist jedoch nicht signifikant $(p=.16)$.

\section{Diskussion}

In dem vorliegenden Beitrag wurde der Frage nachgegangen, wie Schülerinnen und Schüler mit ihren individuellen heterogenen Verhaltensweisen Peerinteraktionen im Unterricht in Abhängigkeit des behavioralen Klassenumfeldes erleben. Hierzu wurden zunächst mittels LPA homogene Subgruppen innerhalb der Schülerschaft identifiziert. Dieser methodische Ansatz entspricht einer personzentrierten Herangehensweise zur Analyse von Erlebens- und Verhaltensauffälligkeiten. Anstatt einer Unterteilung der Jugendlichen in Kategorien anhand von Cut-Off-Werten oder diagnostizierter Verhaltensauffälligkeiten wurde in der vorliegenden Studie ein dimensionaler Ansatz verfolgt, um der gesamten Schülerschaft zu entsprechen.

Generell deuten die Befunde darauf hin, dass die Schülerinnen und Schüler der fünften und sechsten Jahrgangsstufe (Ende Primarstufe) in Unterrichtssituationen, in denen sie mit ihren Peers arbeiteten, sowohl engagierter und motivierter als auch weniger gestresst und weniger verärgert sind. Diese Effekte sind für Schülerinnen und Schüler mit internalisierenden oder externalisierenden Verhaltens- weisen ausgeprägter als für diejenigen mit adaptivem Verhalten. Interaktionen mit Peers können demnach als eine bedeutsame Quelle für aktuelle Motivation im Unterricht erachtet werden - unabhängig vom Profiltyp. Demzufolge eignen sich Partner- oder Gruppenarbeiten sowohl zur Steigerung der Motivation und des Interesses am Unterricht sowie zum Abbau von Stress und Ängsten im Unterricht für Schülerinnen und Schüler der gesamten Schülerschaft, insbesondere für diejenigen mit internalisierendem oder externalisierendem Verhalten. Dieser Befund entspricht im Wesentlichen den Ergebnissen einer Untersuchung in der achten und neunten Jahrgangsstufe (Ende Sekundarstufe I), in der ebenfalls eine hohe positive und eine tiefe negative Aktivierung während sozialer Peerinteraktionen für Jugendliche mit und ohne sonderpädagogischem Unterstützungsbedarf berichtet wird (Zurbriggen et al., 2018). In diesem Zusammenhang kann gemäß der Selbstbestimmungstheorie (Ryan \& Deci, 2000) angenommen werden, dass Peerinteraktionen im Unterricht zur Befriedigung des Grundbedürfnisses nach sozialer Eingebundenheit beitragen und dementsprechend Partneroder Gruppenarbeiten mit erhöhter Motivation einhergehen. In der Schulpraxis erscheint es daher erstrebenswert, begünstigende Bedingungen für soziale Interaktionen herzustellen und Kontakte zu Peers zu ermöglichen, um bei Schülerinnen und Schülern ein positives emotionales Erleben anzuregen. Neben der Etablierung von Helfer-Systemen im Klassenraum, können jegliche Unterrichtsformen (wie etwa Gruppenpuzzle oder Museumsrundgänge), die 
den Austausch der Schülerinnen und Schüler untereinander fördern und ermöglichen, als Möglichkeiten der Motivationsförderung eingesetzt werden.

Es kann vermutet werden, dass soziale Interaktionen im schulischen Kontext stärker von außen gesteuert und initiiert werden. Daher profitieren insbesondere Jugendliche mit internalisierenden oder externalisierenden Verhaltensweisen, die sonst häufig Schwierigkeiten im Knüpfen und Aufrechterhalten von freundschaftlichen Beziehungen zu Gleichaltrigen (Lauer \& Renk, 2013; Olympia et al., 2013), im Allgemeinen weniger Kontakte zu Peers haben und in ihren Klassen häufig sozial ausgegrenzt werden, im Vergleich zu ihren Peers mit adaptivem Verhalten stärker von Partner- oder Gruppenarbeiten.

Die geringfügigen, jedoch nicht signifikanten differenziellen moderierenden Effekte verweisen darauf, dass der Zusammenhang des emotionalen Erlebens und der Peerinteraktionen für Jugendliche nicht durch ihr Klassenumfeld in Bezug auf prosoziales oder problematisches Verhalten moderiert wird. Die relativ kleine Stichprobe auf Klassenebene $(N=40)$ und die geringe klassenbedingte Varianz könnten das Ausbleiben eines signifikanten Effekts erklären. Entgegen der theoretischen Annahmen ist daher der Effekt sozialer Interaktionen auf das emotionale Erleben in allen untersuchten Klassen - unabhängig des behavioralen Klassenumfeldes - ähnlich hoch. Möglicherweise werden soziale Interaktionen in prosozialen Klassen als positiv erlebt, weil diese alltäglich vorkommen und Jugendliche dieser Klassen Regeln und Gepflogenheiten von Interaktionen kennen und anwenden. Aber auch Lernende in verhaltensproblematischeren Klassen erleben Interaktionen mit ihren Peers als motivierend. Insofern kann dieser Befund insbesondere für Klassen mit verhaltensproblematischen Lernumfeldern als Chance verstanden werden, da Interaktionen mit Peers auch in diesen Klassen als positiv wahrgenommen werden, und das emotionale Erleben der Schülerinnen und Schüler so angeregt werden kann. Grundsätzlich scheint somit das Streben nach sozialer Eingebundenheit als psychologisches Grundbedürfnis für Jugendliche in unterschiedlichsten behavioralen Lernumfeldern zu gelten.

\section{Limitationen und Ausblick}

Im Rahmen dieses Beitrags wurden latente Profile basierend auf Ratings von Lehrpersonen gebildet. Allerdings bestehen zwischen den Einschätzungen von Lehrpersonen und von Schülerinnen und Schülern relativ große Diskrepanzen (z.B. Gomez, 2014). Insbesondere internalisierendes Problemverhalten wird von Lehrpersonen weitaus seltener identifiziert als aus der Perspektive der
Jugendlichen berichtet wird (Lüdeke \& Linderkamp, 2018). Auch hinsichtlich der emotionalen und sozialen Inklusion von Schülerinnen und Schülern liegen Ergebnisse vor, die auf eine eher geringe Übereinstimmung zwischen der Selbstsicht und der Perspektive ihrer Lehrpersonen deuten (Venetz, Zurbriggen \& Schwab, 2019). Generell empfiehlt es sich daher, verschiedene Perspektiven zu berücksichtigen, da diesen unterschiedliche Wahrnehmungsprozesse zugrunde liegen. Zudem können zur detaillierteren Identifizierung latenter Profile in Bezug auf heterogen Erlebens- und Verhaltensweisen weitere relevante Merkmale wie das Geschlecht oder das Alter der Jugendlichen herangezogen werden.

Es konnten ausschließlich marginale, jedoch nicht signifikante, moderierende Kompositionseffekte in Bezug auf das Verhalten der Jugendlichen identifiziert werden. Möglicherweise kann dieser Umstand durch die Operationalisierung der Klassenkomposition in Form von individuellen Umgebungswerten basierend auf subjektiven Verhaltenseinschätzungen durch Lehrpersonen begründet werden. Zur Ermittlung des behavioralen Klassenumfeldes bietet sich ebenfalls die Berücksichtigung mehrerer Perspektiven, wie etwa Beobachtungsverfahren durch objektive Rater oder Selbstberichtsverfahren für Schülerinnen und Schüler, an. Signifikante Kompositionseffekte auf der Ebene der Schule für sozial-emotionale Merkmale von Jugendlichen der neunten Klasse (Nikolov \& Dumont, 2020) deuten zudem darauf hin, dass die Berücksichtigung der Zusammensetzung der Schülerinnen und Schüler auf Schulebene ebenfalls relevant für die Überprüfung von Moderationseffekten sein kann.

Insbesondere bei mehrebenenanalytischen Verfahren werden große Stichproben auf den verschiedenen Ebenen benötigt, um bedeutsame Effekte zu identifizieren und aussagekräftige Schlussfolgerungen ableiten zu können. Zwar ist die vorliegende Stichprobe mit 8870 Momentaufnahmen von 719 Schülerinnen und Schüler für die Forschungsfragen dieses Beitrags ausreichend, um weiterführend aber auch Klasseneffekte modellieren zu können, sind mehr als die vorliegenden 40 Klassen wünschenswert.

Bei der Interpretation der Ergebnisse dieses Beitrags muss berücksichtigt werden, dass es sich bei der in dieser Studie verwendeten ESM zwar per Definition um eine intensive longitudinal method (Bolger \& Laurenceau, 2013) handelt, die jedoch „nur“ fünf aufeinanderfolgende Schultage umfasst. Um kausale Einflüsse sozialer Interaktionen auf das emotionale Erleben von Jugendlichen in Abhängigkeit ihres behavioralen Klassenumfeldes bestätigen zu können, sind längsschnittliche Untersuchungen mit mehreren ESM-Phasen über einen größeren Zeitraum erforderlich (sogenannte measurement-burst designs; z.B. Röcke, Hoppmann \& Klumb, 2011; Sliwinski, Almeida, Smyth \& Stawski, 2009). 
In dieser Untersuchung wurde ausschließlich ein Aspekt sozialer Interaktionen - nämlich die wahrgenommene Qualität in Form des emotionalen Erlebens - erfasst. So liegen beispielsweise keine Informationen über die Dauer der Interaktion oder die körperliche Nähe des Interaktionspartners bzw. der Interaktionspartnerin vor oder darüber, ob die Peerinteraktion von der Lehrperson gesteuert wurde. Neben einer Erweiterung der ESM-Befragung zur genaueren Rekonstruktion der sozialen Interaktion im Unterricht ist etwa der Einsatz von sogenannten RFID (radio-frequency identification)-Badges möglich, mit denen die Dauer und Nähe sozialer Interaktionen zwischen Schülerinnen und Schülern objektiv gemessen und aufgezeichnet wird (siehe hierzu z. B. Elmer, Chaitanya, Purwar \& Stadtfeld, 2019). Die Kombination von RFID- und ESMMessungen erfolgte bereits im Rahmen eines kürzlich durchgeführten Pilotprojekts. Die Zusammenführung beider Messungen lässt auf aufschlussreiche Erkenntnisse in Bezug auf soziale Interaktionen im Unterricht hoffen. Zukünftig sind weitere empirische Studien wünschenswert, die verschiedene Ansätze zur Operationalisierung und Analyse sozialer Interaktionen verfolgen (für einen Überblick Zurbriggen, 2018b), wie etwa soziale Netzwerkanalysen (z. B. Kulawiak \& Wilbert, 2019; Zander, Chen \& Hannover, 2019), und dadurch ein vertieftes Verständnis von sozial-emotionalen Einflussprozessen zwischen Peers ermöglichen.

\section{Literatur}

Barth, J. M., Dunlap, S. T., Dane, H., Lochman, J. E. \& Wells, K. C. (2004). Classroom environment influences on aggression, peer relations, and academic focus. Journal of School Psychology, 42, $115-133$

Bellmore, A. D., Witkow, M. R., Graham, S. \& Juvonen, J. (2004). Beyond the individual: The impact of ethnic context and classroom behavioral norms on victims' adjustment. Developmental Psychology, 40 (6), $1159-1172$.

Besser, A., Döhnert, M. \& Stadelmann, S. (2019). Verschiedene sozioökonomische Faktoren als Prädiktoren für internalisierende und externalisierende Störungsbilder des Kindes- und Jugendalters. Zeitschrift für Kinder- und Jugendpsychiatrie und Psychotherapie, 47 (4), 345-358. https://doi.org/10.1024/14224917/a000668

Bilz, L. (2008). Schule und psychische Gesundheit. Risikobedingungen für emotionale Auffälligkeiten von Schülerinnen und Schülern. Wiesbaden: Springer.

Bolger, N. \& Laurenceau, J.-P. (2013). Intensive longitudinal methods. An introduction to diary and experience sampling research. New York: The Guilford Press.

Bosacki, S., Dane, A., Marini, Z. \& YLC-CURA (2007). Peer relationships and internalizing problems in adolescents: Mediating role of self-esteem. Emotional and Behavioural Difficulties, 12 (4), 261 - 282. https://doi.org/10.1080/13632750701664293

Chen, F. F. (2007). Sensitivity of goodness of fit indexes to lack of measurement invariance. Structural Equation Modeling: A Multi- disciplinary Journal, 14 (3), 464-504. doi:10.1080/1070 5510701301834

Dai, D. Y. \& Sternberg, R. J. (2004). Motivation, emotion, and cognition: Integrative perspectives on intellectual functioning and development. Mahwah, New Jersey: Lawrence Erlbaum Associates.

Dishion, T. J. \& Tipsord, J. M. (2011). Peer contagion in child and adolescent social and emotional development. Annual Review of Psychology, 62, $189-214$.

Elmer, T., Chaitanya, K., Purwar, P. \& Stadtfeld, C. (2019). The validity of RFID badges measuring face-to-face interactions. Behavior Research Methods, 51,1-19.

Gerrig, R. J. \& Zimbardo P. G. (2008). Psychologie (18., aktualisierte Aufl.). München: Pearson.

Goetz, T., Preckel, F., Pekrun, R. \& Hall, N. C. (2007). Emotional experiences during test taking: Does cognitive ability make a difference? Learning and Individual Differences, 17, 3-16.

Gomez, R. (2014). Correlated trait-correlated method minus one analysis of the convergent and discriminant validities of the Strengths and Difficulties Questionnaire. Assessment, 21 (3), $372-382$.

Goodman, R. (1997). The Strengths and Difficulties Questionnaire: A research note. Journal of Child Psychology and Psychiatry, 38 (5), $581-586$.

Groen, G., Pössel, P. \& Petermann, F. (2004). Depression im Kindes- und Jugendalter. In F. Petermann, K. Niebank \& H. Scheithauer (Hrsg.), Entwicklungswissenschaft (S.437-481). Berlin: Springer.

Hascher, T. \& Brandenberger, C. C. (2018). Emotionen und Lernen im Unterricht. In M. Huber \& S. Krause (Hrsg.), Bildung und Emotion (S. 289 -310). Wiesbaden: Springer.

Hektner, J. M., Schmidt, J. A. \& Csikszentmihalyi, M. (2007). Experience sampling method. Measuring the quality of everyday life. Thousand Oaks: Sage Publications.

Hoglund, W. L. \& Leadbeater, B. J. (2004). The effects of family, school, and classroom ecologies on changes in children's social competence and emotional and behavioral problems in first grade. Developmental Psychology, 40 (4), $533-544$.

Hox, J. J. (2010). Multilevel analysis: Techniques and applications (2nd ed.). New York, NY: Routledge.

Hu, L. \& Bentler, P. M. (1999). Cutoff criteria for fit indexes in covariance structure analysis: Conventional criteria versus new alternatives. Structural Equation Modeling: A Multidisciplinary Journal, 6 (1), $1-55$.

Izard, C. E. (1999). Die Emotionen des Menschen. Eine Einführung in die Grundlagen der Emotionspsychologie. Weinheim: Beltz.

Krawinkel S., Südkamp, A., Lange, S. \& Tröster, H. (2017). Soziale Partizipation in inklusiven Grundschulklassen: Bedeutung von Klassen- und Lehrkraftmerkmalen. Empirische Sonderpädagogik, 3, 277-295.

Kulawiak, P.R. \& Wilbert, J. (2019). Introduction of a new method for representing the sociometric status within the peer group: The example of sociometrically neglected children. International Journal of Research \& Method in Education. https://doi.org/10.1 080/1743727X.2019.1621830.

Kullmann, H., Geist, S. \& Lütje-Klose, B. (2015). Erfassung schulischen Wohlbefindens in inklusiven Schulen - Befunde zur Erprobung eines mehrdimensionalen Konstrukts in fünfJahrgängen der Sekundarstufe I an der Laborschule Bielefeld. In P. Kuhl, P. Stanat, B. Lütje-Klose, C. Gresch, H. Anand Pant \& M. Prenzel (Hrsg.), Inklusion von Schülerinnen und Schülern mit sonderpädagogischem Förderbedarf in Schulleistungserhebungen (S.301-333). Wiesbaden: Springer/VS.

La Greca, A. \& Harrison, H. (2005). Adolescent peer relations, friendships, and romantic relationships: Do they predict social anxiety and depression. Journal of Clinical Child and Adolescent Psychology, 34, 49-61. 
Laird, R. D., Jordan, K. Y., Dodge, K. A., Pettit, G. S. \& Bates, J. E. (2001). Peer rejection in childhood, involvement with antisocial peers in early adolescence, and the development of externalizing behavior problems. Development and Psychopathology, 13 (2), $337-354$.

Lane, K. L., Oakes, W. P., Menzies, H. M. \& Germer, K. A. (2014). Screening and identification approaches for detecting students at risk. In H. M. Walker \& F. M. Gresham (Eds.), Handbook of evidence-based practices for students having emotional and behavioral disorders (pp.129-151). New York, NY: Guilford.

Lauer, B. M. \& Renk, K. (2013). The peer informant: Characteristics related to the perceptions of peer behavior problems. Journal of Child and Family Studies, 22 (6), $786-800$.

Lo, Y., Mendell, N. R. \& Rubin, D. B. (2001). Testing the number of components in a normal mixture. Biometrika, 88, 767-778.

Lüdeke, S. (2018). Verhaltensprobleme im Jugendalter. Wiesbaden: Springer.

Lüdeke, S. \& Linderkamp, F. (2018). Beurteilerdiskrepanzen als Indikatoren für Schulstress. Eine Studie zu Einschätzungen internalisierender und externalisierender Verhaltensprobleme aus Sicht von Jugendlichen und Lehrpersonen. Empirische Sonderpädagogik, 10 (4), $353-369$.

Lyubomirsky, S., King, L. \& Diener, E. (2005). The benefits of frequent positive affect: Does happiness lead to success? Psychological Bulletin, 131 (6), $803-855$.

Morin, A.J.S. \& Marsh, H. W. (2015). Disentangling shape from level effects in person-centered analyses: An illustration based university teachers' multidimensional profiles of effectiveness. Structural Equation Modeling: A Multidisciplinary Journal, 22 (1), 39 - 59. doi:10.1080/10705511.2014.919825

Mercer, S. H., McMillen, J. S. \& DeRosier, M. E. (2009). Predicting change in children's aggression and victimization using classroom-level descriptive norms of aggression and pro-social behavior. Journal of School Psychology, 47 (4), 267 - 289.

Müller, C. M. \& Zurbriggen, C. L. A. (2016). An overview of classroom composition research on social-emotional outcomes: Introduction to the Special Issue. Journal of Cognitive Education and Psychology, 15 (2), $163-184$.

Muthén, L. K. \& Muthén, B. O. (1998 - 2017). Mplus user's guide (7th ed.). Los Angeles, CA: Muthén \& Muthén.

Nikolov, F. \& Dumont, H. (2020). Das Ganze ist mehr als die Summe seiner Teile: Schulkomposition, Schulzufriedenheit und normverletzendes Verhalten. Journal für Bildungsforschung Online, $12(1), 26-46$.

Olympia, D. E., Heathfield, L. T., Jenson, W. R., Majszak, H., RamosMatias, V. \& Thacker, M. (2013). Positive psychology and children with emotional and behavioral difficulties. In M. L. Wehmeyer (Ed.), The Oxford handbook of positive psychology and disability (pp. 475 - 493). Oxford: Oxford University Press.

Papandrea, K. \& Winefield, H. (2011). It's not just the squeaky wheels that need the oil: Examining teachers' views on the disparity between referral rates for students with internalizing versus externalizing problems. School Mental Health, 3 (4), $222-235$.

Parker, P. D., Ciarrochi, J., Heaven, P., Marshall, S., Sahdra, B. \& Kiuru, N. (2015). Hope, friends, and subjective well-being: A social network approach to peer group contextual effects. Child Development, 86 (2), 642 - 650. https://doi.org/10.1111/cdev.12308

Pekrun, R. \& Frenzel, A. C. (2009). Persönlichkeit und Emotion. In V. Brandstätter \& J. H. Otto (Hrsg.), Handbuch der Allgemeinen Psychologie - Motivation und Emotion (S.686 - 696). Göttingen: Hogrefe.

Pekrun, R. \& Linnenbrink-Garcia, L. (2014). International handbook of emotions in education. New York: Routledge.

Prinstein, M. J. (2007). Moderators of peer contagion: A longitudinal examination of depression socialization between adoles- cents and their best friends. Journal of Clinical Child and Adolescent Psychology, 36 (2), $159-170$.

Reisenzein, R. (2018). Emotionen in Lern- und Leistungskontexten. In B. Spinath, O. Dickhäuser \& C. Schöne (Hrsg.), Psychologie der Motivation und Emotion - Grundlagen und Anwendung in ausgewählten Lern- und Arbeitskontexten (S.80 - 107). Göttingen: Hogrefe.

Röcke, C., Hoppmann, C.A. \& Klumb, P.L. (2011). Correspondence between retrospective and momentary ratings of positive and negative affect in old age: Findings from a one-year measurement burst design. The Journals of Gerontology, Series B: Psychological Sciences and Social Sciences, 66 (4), 411 - 415. https://doi. org/10.1093/geronb/gbr024

Rubin, K. H., Bukowski, W. \& Parker, J. (2006). Peer interactions, relationships, and groups. In N. Eisenberg (Ed.), Social, Emotional, and Personal Development (pp. 571 -645). New York: Wiley.

Ryan, R. M. \& Deci, E. L. (2000). Self-determination theory and the facilitation of intrinsic motivation, social development, and well-being. American Psychologist, 55, 68-78. https://doi. org/10.1037/0003-066X.55.1.68

Schallberger, U. (2005). Kurzskala zur Erfassung der positiven Aktivierung, negativen Aktivierung und Valenz in Experience Sampling Studien (PANAVA-KS). Forschungsbericht. Zürich: Psychologisches Institut, Universität Zürich.

Scherer, K. R. (2009). The dynamic architecture of emotion: Evidence for the component process model. Cognition and Emotion, 23 (7), 1307 - 1351. https://doi.org/10.1080/026999309029 28969

Scherreiks, L. \& Schwalbe, A. (2019). Internalisierende Auffälligkeiten in der Schule erkennen. Potsdamer Zentrum für empirische Inklusionsforschung, 1,1-9.

Schmidt-Atzert, L. (2009). Kategoriale und dimensionale Modelle. In V. Brandstätter \& J. H. Otto (Hrsg.), Handbuch der Allgemeinen Psychologie - Motivation und Emotion (S.571 - 576). Göttingen: Hogrefe.

Schulte-Köme, G. (2016). Psychische Störungen bei Kindern und Jugendlichen im schulischen Umfeld. Deutsches Ärzteblatt, $113(11), 183-191$

Sherif, M. \& Hovland, C. I. (1961). Social judgment: Assimilation and contrast effects in communication and attitude change. Oxford, UK:Yale University Press.

Sliwinski, M. J., Almeida, D. M., Smyth, J. \& Stawski, R. S. (2009). Intraindividual change and variability in daily stress processes: Findings from two measurement-burst diary studies. Psychology and Aging, 24 (4), $828-840$.

Steffen, A., Akmatov, M. K., Holstiege, J. \& Bätzing, J. (2018). Diagnoseprävalenz psychischer Störungen bei Kindern und Jugendlichen in Deutschland: Eine Analyse bundesweiter vertragsärztlicher Abrechnungsdaten der Jahre 2009 bis 2017. Zentralinstitut für die assenärztliche Versorgung in Deutschland (Zi). Versorgungsatlas-Bericht Nr. 18/07. Berlin. https://doi.org/10.20364/ VA-18.07

Stone, A. A. \& Litcher-Kelly, L. (2006). Momentary capture of realworld data. In M. Eid \& E. Diener (Eds.), Multimethod Measurement in Psychology (pp. 61 - 72). Washington, DC: American Psychological Association.

Venetz, M. (2015). Trend: Schulische Integration und Wohlbefinden von Kindern und Jugendlichen mit besonderem Förderbedarf. Vierteljahresschrift für Heilpädagogik und ihre Nachbargebiete, 84 (1), $57-59$.

Venetz, M., Tarnutzer, R., Zurbriggen, C. \& Sempert, W. (2012). Emotionales Erleben im Unterricht und schulbezogene Selbstbilder. Vergleichende Analysen von Lernenden in integrativen und separativen Schulformen. Bern: Edition SZH / CSPS.

Venetz, M. \& Zurbriggen, C. (2015). Intensive Longitudinal Methods - ihre Eignung für die sonderpädagogische Forschung und ex- 
emplarische Anwendungsmöglichkeiten. Empirische Sonderpädagogik, 7 (3), $194-205$.

Venetz, M. \& Zurbriggen, C. (2016). Intensity Bias oder Rosy View? Zur Diskrepanz habituell und aktuell berichtetem emotionalen Erleben im Unterricht. Empirische Pädagogik, 30 (1), 27 - 42.

Venetz, M., Zurbriggen, C. L. A. \& Schwab, S. (2019). What do teachers think about their students' inclusion? Consistency of students' self-reports and teacher ratings. Frontiers in Psychology, 10, 1637. https://doi.org/10.3389/fpsyg.2019.01637

Watson, D. \& Tellegen, A. (1985). Toward a consensual structure of mood. Psychological Bulletin, 98, 219- 235.

Watson, D., Wiese, D. Vaidya, J. \& Tellegen, A. (1999). The two general activation systems of affect: Structural findings, evolutionary considerations, and psychobiological evidence. Journal of Personality and Social Psychology, 76 (5), $98-112$.

Zander, L., Chen, I. \& Hannover, B. (2019). Who asks whom for help in mathematics? A sociometric analysis of adolescents' helpseeking within and beyond clique boundaries. Learning and Individual Differences, 72, 49 - 58. https://doi.org/10.1016/j.lindif. 2019.03.002

Zurbriggen, C. (2018a). Dabei oder nicht dabei? Soziale Partizipation im Jugendalter. Sonderpädagogische Förderung heute, 61 (4), $407-416$.

Zurbriggen, C. (2018b). Methoden der empirischen Erforschung von Beziehungen und Einflussprozessen zwischen Klassenkameradinnen und -kameraden. Vierteljahresschrift für Heilpädagogik und ihre Nachbargebiete, 87 (3), 205 -217. https://doi. org/10.2378/vhn2018.art22d

Zurbriggen, C. \& Venetz, M. (2016). Soziale Partizipation und aktuelles Erleben im gemeinsamen Unterricht. Empirische Pädagogik, 30, $98-112$.

Zurbriggen, C. \& Venetz, M. (2018). Diversität und aktuelles emotionales Erleben von Schülerinnen und Schülern im inklusiven Unterricht. In G. Hagenauer \& T. Hascher (Hrsg.), Emotionen und Emotionsregulation in Schule und Hochschule (S.87-102). Münster:Waxmann.
Zurbriggen, C. L. A., Venetz, M. \& Hinni, C. (2018). The quality of experience of students with and without special educational needs in everyday life and when relating to peers. European Journal of Special Needs Education, 1-16. https://doi.org/10.1 080/08856257.2018.1424777

\section{Historie}

Manuskript eingereicht: 28.11.2019

Manuskript nach Revision angenommen: 13.10.2020

Onlineveröffentlichung: 16.11.2020

\section{Danksagung}

Wir danken und gedenken Prof. Dr. Martin Venetz, der bei einer Vorversion für eine Tagungspräsentation maßgeblich mitgewirkt hatte.

\section{Förderung}

Das Projekt, auf dessen Datengrundlage dieser Beitrag basiert, wurde vom Schweizerischen Nationalfonds und der Interkantonalen Hochschule für Heilpädagogik Zürich finanziert.

\section{ORCID}

Margarita Knickenberg

https://orcid.org/0000-0001-5521-0417

\section{Dr. Margarita Knickenberg}

Universität Bielefeld

Fakultät für Erziehungswissenschaft

Arbeitsbereich Erziehungswissenschaft mit dem Schwerpunkt erschwerte Lern- und Entwicklungsbedingungen

Universitätsstr. 25

33615 Bielefeld

margarita.knickenberg@uni-bielefeld.de 